\title{
Nucleation in binary polymer blends: A self-consistent field study
}

\author{
Stephen M. Wood and Zhen-Gang Wang \\ Division of Chemistry and Chemical Engineering, California Institute of Technology, Pasadena, \\ California 91125
}

(Received 30 July 2001; accepted 31 October 2001)

\begin{abstract}
We study the structure and thermodynamics of the critical nuclei in metastable binary polymer blends using the self-consistent field method. At the mean-field level, our results are valid throughout the entire metastable region and provide a smooth crossover from the classical capillary-theory predictions near the coexistence curve to the density functional predictions of Cahn and Hilliard (properly transcribed into expressions involving the parameters of the binary polymer blends) near the spinodal. An estimate of the free energy barrier provides a quantitative criterion (the Ginzburg criterion) for the validity of the (mean-field) self-consistent approach. The region where mean-field theory is valid and where there can be a measurable nucleation rate is shown to be poorly described by the existing limiting theories; our predictions are therefore most relevant in this region. We discuss our results in connection with recent experimental observations by Balsara and co-workers. () 2002 American Institute of Physics. [DOI: 10.1063/1.1429956]
\end{abstract}

\section{INTRODUCTION}

The formation of a new phase from a homogeneous metastable state occurs through an activated process known as nucleation. ${ }^{1}$ Although nucleation is fundamentally a nonequilibrium phenomenon, if the time scale for nucleation is sufficiently long relative to the molecular relaxation time, the transition state - the critical nucleus - can be considered as a quasiequilibrium state, leading to an Arrhenius-type expression for the nucleation rate $J=J_{0} \exp (-\Delta F / k T)$, where $J_{0}$ is a kinetic prefactor associated with molecular relaxation and $\Delta F$ is the free energy of formation of the critical nucleus. ${ }^{1,2}$ Because of the exponential dependence, the rate of nucleation is largely determined by $\Delta F$, which depends strongly on the thermodynamic state of the metastable system, e.g., the distance from the equilibrium coexistence curve. On the other hand, the kinetic prefactor is relatively insensitive to the thermodynamic state. Therefore, determination of the critical nucleus and its free energy of formation is a central problem for a molecular theory of nucleation.

In this work, we consider nucleation in a metastable binary polymer blend. In a seminal paper that provided much insight into the dynamics of binary polymer blends, Binder ${ }^{3}$ examined the problem of nucleation in a metastable binary blend by combining results from the classical capillary theory (hereafter referred to as the classical theory) near the coexistence curve and from the Cahn-Hilliard theory for binary mixtures ${ }^{4}$ near the spinodal (hereafter referred to as the Cahn-Hilliard asymptotic theory). Close to the coexistence curve, the classical theory predicts a large nucleation barrier that increases to infinity as the composition/temperature approaches the coexistence value. While classical theory provides an accurate description of the critical nucleus near the coexistence curve, its predictions are essentially irrelevant because the rate of nucleation in this region is unobservably small for typical degrees of polymerization. The applicability of the Cahn-Hilliard asymptotic theory is similarly limited but for different reasons. While yielding fundamental insights into the nature of the spinodal and becoming increasingly accurate (in the mean-field sense) as the spinodal is approached, the predicted nucleation barrier becomes meaningless when the composition/temperature is too close to the spinodal due to the large thermal fluctuation. ${ }^{5}$ Thus as far as nucleation in a binary blend is concerned, the experimentally most relevant range of composition/temperature is probably in the broad crossover region between these two limits, for which no theoretical studies have been conducted explicitly on polymer blends. Recent experiments by Balsara and co-workers ${ }^{6-8}$ suggest the inadequacy of existing theories and point to the need for a more systematic theoretical examination of nucleation in polymeric systems.

In this paper, we present results of a self-consistent field study of nucleation in a binary polymer blend. At the meanfield level, self-consistent field theory (SCF) provides a systematic and accurate description of inhomogeneous polymeric fluids, as it yields detailed information on the chain conformation, structure and thermodynamics. SCF has been used successfully to study interfaces in polymer blends and solutions, ${ }^{9}$ polymer adsorption at surfaces, ${ }^{10}$ and microphase separation in block copolymers. ${ }^{11,12}$ The SCF can be considered the polymeric counterpart of the density-functional approach for small-molecule systems, which has met with considerable success in the study of nucleation in simple fluids. ${ }^{2,13}$ In this regard, we note that even a systematic study of nucleation in binary polymer blends using the approximate Flory-Huggins-de Gennes square-gradient free energy functional approach does not yet exist in the literature. The SCF bypasses any approximations with regard to spatial correlations (such as the square-gradient approximation) because the chain connectivity is fully accounted for. Therefore, at the mean-field level, the predictions of SCF are valid throughout the entire metastable region and provide a smooth crossover from the behavior near the coexistence curve to that near the spinodal. SCF also provides a consis- 
tency criterion for its own validity, through a quantitative evaluation of the nucleation barrier near the spinodal. ${ }^{5}$ Thus a SCF study serves as an important benchmark for evaluating the accuracy of approximate theories, for assessing the validity of the mean-field approximation, and for suggesting further improvements in the theoretical understanding of nucleation in polymeric systems.

The rest of this paper is organized as follows: in Sec. II, we provide the essential SCF equations for a binary polymer blend that are used for determining the density profile of the critical nucleus and its free energy of formation. In Sec. III, we present and discuss the results of our SCF study. For a chosen composition, we calculate the density profiles of the critical nuclei and the free energy of formation as we move from the coexistence curve to the spinodal. From the profile of the critical nucleus, we obtain the nucleus size, as well as the excess material contained within the nucleus. Our results are compared with predictions of the classical theory near the coexistence curve using the interfacial free energy that we have calculated for a flat interface. Near the spinodal, we compare our results with those of the Cahn-Hilliard asymptotic theory by a proper transcription of their results into parameters for the polymer blend using the FloryHuggins-de Gennes free energy functional. Our results agree with the respective theories in these two limits. We then discuss the validity of the SCF theory in conjunction with recent experimental results of Balsara and co-workers. Section IV summarizes the main findings of this paper. Finally, we provide an Appendix that contains detailed derivation of some of the theoretical results used in the paper.

\section{SELF-CONSISTENT FIELD THEORY}

We consider an incompressible binary $A / B$ polymer blend. Insofar as nucleation represents a spatially localized fluctuation, we may focus on a subvolume $V$ of the entire system. Because the system is incompressible, we may choose the volume $V$ and the volume fraction of one of the polymers, say $A$, as the independent thermodynamic variables. Henceforth, for notational conciseness, we will use $\phi$ to denote the volume fraction of the $A$ polymers; the volume fraction of the $B$ polymers is simply $1-\phi$. Since the volume $V$ is part of a larger system, it is convenient to treat $V$ as an open system in equilibrium with a homogeneous reservoir at composition $\phi_{0}$. The appropriate free energy is the grand potential, defined as

$$
G=\int d \mathbf{r}\left(f[\phi(\mathbf{r})]-\mu_{0} \phi(\mathbf{r})\right),
$$

where $f$ is the Helmholtz free energy density of the system and $\mu$ is a chemical potential-like variable that is conjugate to the volume fraction $\phi$. For a uniform system, $\mu$ is defined as

$$
\mu \equiv \frac{\partial f}{\partial \phi} .
$$

The subscript 0 on $\mu$ in Eq. (2.1) means that this quantity is evaluated at the homogeneous bulk composition $\phi_{0}$. For a given specification of $\mu_{0}$, the equilibrium profile $\phi(\mathbf{r})$ is obtained by a functional minimization of the free energy $G$. The critical nucleus corresponds to a saddle point of the free energy functional; it is a maximum with respect to the exchange of species in the volume with that in the reservoir, but a minimum with respect to the density profiles for a given overall composition in the volume.

Once the density profile $\phi(\mathbf{r})$ is determined, the excess free energy with respect to the homogeneous state is then calculated as

$$
\begin{aligned}
\Delta F & =G-G_{0} \\
& \equiv \int d \mathbf{r}\left(f[\phi(\mathbf{r})]-f\left(\phi_{0}\right)-\mu_{0}\left(\phi(\mathbf{r})-\phi_{0}\right)\right) .
\end{aligned}
$$

This is the free energy change associated with creating a density profile $\phi(\mathbf{r})$ out of a large, uniform system at composition $\phi_{0}$. For the critical nucleus, this is then the reversible work of formation, or the free energy barrier for nucleation. In this paper, these two terms will be used interchangeably.

The free energy functional $G$ or $\Delta F$ is not available analytically for arbitrary inhomogeneity. A common approximation is to combine the Flory-Huggins free energy for a homogeneous blend with nonlocal terms represented by the square of the density gradients, as first proposed by de Gennes. ${ }^{14,15}$ Such an approximation is valid for weak inhomogeneity but becomes inaccurate when the length scale of spatial variation becomes comparable to the radius of gyration of the polymers. ${ }^{3,16,17}$ We avoid making any such approximations introduced in constructing an analytical free energy by resorting to a numerical self-consistent field theory which, though still mean-field in nature, accounts fully the spatial correlations due to the chain connectivity.

The SCF theory treats the many chain problem as an effective single polymer chain in a field that is to be determined self-consistently. A systematic derivation of the selfconsistent field equations for polymer blends was given by Noolandi and Hong ${ }^{9}$ using a canonical formulation. For the nucleation problem at hand, it is more convenient to formulate the SCF using a grand canonical ensemble. The details of the derivation are provided in the Appendix. Here we summarize the key equations.

For an incompressible $A / B$ binary polymer blend, with degrees of polymerization $N_{A}$ and $N_{B}$, monomer volumes $v_{A}$ and $v_{B}$, and Kuhn lengths $b_{A}$ and $b_{B}$ for the two respective polymers, the grand free energy of the system in equilibrium with a bulk reservoir of composition $\phi_{0}$ and chemical potential $\mu_{0}$ is

$$
\begin{aligned}
\beta G= & \int d \mathbf{r}\left[\chi \phi(1-\phi)-w_{A} \phi-w_{B}(1-\phi)\right] \\
& -\frac{\exp \left(\beta \mu_{0} N_{A} v_{A}\right) Z_{A}\left(w_{A}\right)}{N_{A} v_{A}}-\frac{Z_{B}\left(w_{B}\right)}{N_{B} v_{B}},
\end{aligned}
$$

where $\chi$ is the Flory-Huggins parameter characterizing the effective repulsion between the two polymer component.

In this equation, $w_{\alpha}(\alpha=A, B)$ is the effective field for the $\alpha$ chains, and $Z_{\alpha}\left(w_{\alpha}\right)$ is the single chain partition function in the field $k T v_{\alpha} w_{\alpha}$, given by 


$$
Z_{\alpha}=\int d \mathbf{r} q_{\alpha}\left(\mathbf{r}, N_{\alpha}\right)
$$

where $q_{\alpha}\left(\mathbf{r}, N_{\alpha}\right)$ is a reduced partition function for a chain with one end at $\mathbf{r}$ and the other end anywhere in the volume, and is determined by the following diffusion equation:

$$
\left(\frac{\partial}{\partial \tau}-\frac{b_{\alpha}^{2}}{6} \nabla_{\mathbf{r}}^{2}+v_{\alpha} w_{\alpha}(\mathbf{r})\right) q_{\alpha}(\mathbf{r} ; \tau)=0,
$$

with initial condition $q_{\alpha}(\mathbf{r} ; 0)=1$. The density profile $\phi(\mathbf{r})$ and the fields $w_{A}(\mathbf{r})$ and $w_{B}(\mathbf{r})$ are obtained from the following self-consistent equations:

$$
\begin{aligned}
& w_{A}(\mathbf{r})-w_{B}(\mathbf{r})=\chi(1-2 \phi(\mathbf{r})), \\
& \phi(\mathbf{r})=\exp \left(\beta \mu_{0} N_{A} v_{A}\right) \int_{0}^{N_{A}} d \tau q_{A}\left(\mathbf{r}, N_{A}-\tau\right) q_{A}(\mathbf{r}, \tau), \\
& 1-\phi(\mathbf{r})=\int_{0}^{N_{B}} d \tau q_{B}\left(\mathbf{r}, N_{B}-\tau\right) q_{B}(\mathbf{r}, \tau) .
\end{aligned}
$$

The chemical potential for the homogenous bulk reservoir $\mu_{0}$ is shown in the Appendix to be

$$
\beta \mu_{0}=\frac{1}{N_{A} v_{A}} \ln \phi_{0}-\frac{1}{N_{B} v_{B}} \ln \left(1-\phi_{0}\right)+\chi\left(1-2 \phi_{0}\right) .
$$

Thus, for a given reservoir composition $\phi_{0}$, Eqs. (2.7)-(2.9) form a solvable set of equations for $\phi(\mathbf{r}), w_{A}(\mathbf{r})$, and $w_{B}(\mathbf{r})$.

The SCF theory presented thus far is completely general and is applicable to any inhomogeneous binary polymer blend; generalization to include more homopolymer components is straightforward. We now proceed to the problem at hand, namely nucleation in a metastable binary blend. In order to highlight the essential features of the problem without introducing unnecessarily many parameters, in this study we treat the fully symmetric blend with $N_{A}=N_{B}=N, v_{A}$ $=v_{B}=v$, and $b_{A}=b_{B}=b$. Assuming spherical symmetry for the shape of the nuclei, the inhomogeneity is only onedimensional along the radial direction.

As previously stated, the critical nucleus corresponds to a free energy maximum with respect to the material excess (defined as the excess amount of $A$ polymers in the nucleus with respect to the bulk composition) in the volume. This makes it difficult for the numerical methods to converge to the correct solution. One way to circumvent this difficulty is to introduce a constraint that demands a given amount of excess; this would produce a nucleus corresponding to the specified excess and its associated free energy of formation. Alternatively and equivalently, we may specify the value of the density of the $A$ polymers at a given radial distance from the center of the nucleus, and allow the system to find the optimal density profile consistent with this specification. The latter method is numerically more convenient and is adopted in our study. Once we obtain the density profile, we can then easily obtain the material excess and the free energy of formation of the nucleus of this particular size. Varying the value of the specified density allows us to obtain the free energy as a function of the amount of material excess. This information provides a free energy surface as a function of

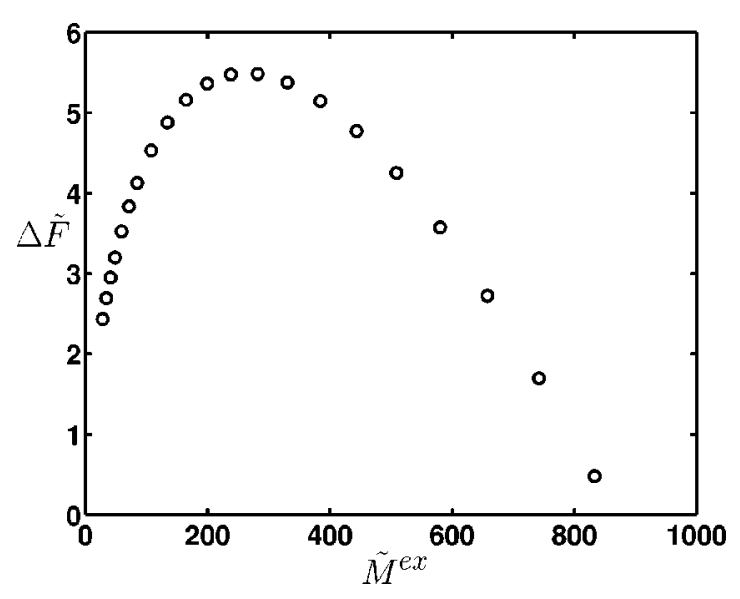

FIG. 1. Free energy of formation of the nucleus as function of material excess, for $\phi_{0}=0.16, N \chi=2.5$. The local maximum in free energy occurs at $\tilde{M}_{\text {crit }}^{\text {ex }}=260$.

the "reaction" coordinates (the amount of excess), the maximum corresponding to the critical nucleus. A typical free energy surface is given in Fig. 1 for a metastable state specified by $\phi_{0}=0.16$ and $N \chi=2.5$. In the remainder of our study, we focus on the properties of the critical nucleus.

\section{RESULTS AND DISCUSSION}

\section{A. Results of the SCF calculation}

We consider a blend consisting of two homopolymers with equal degree of polymerization $N$, monomer volume $v$, and Kuhn length $b$. The mean-field phase behavior is determined by two variables: the volume fraction of the $A$ polymers $\phi$ and the combination $N \chi v$. In the literature, the combination $\chi v$ is customarily called $\chi$. For conciseness, we will use $\chi$ for $\chi v$ in the rest of this paper. The phase diagram is shown in Fig. 2 with a critical point at $(N \chi)_{\text {crit }}=2$. To study nucleation, we take a fixed composition $\phi_{0}$ in the region

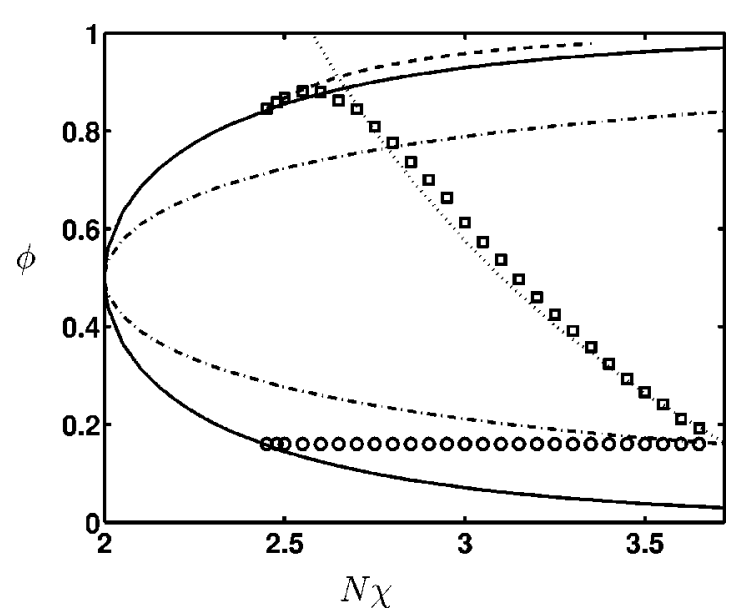

FIG. 2. Density at the center of the critical nucleus as function of $N \chi$. The metastable bulk state is given by the circles, and the density at the center of the nucleus is given by the corresponding squares. The solid curve is the coexistence curve, and the dashed-dotted curve is the spinodal. The dotted curve is the result of Cahn-Hilliard near the spinodal, and the dashed curve is the prediction of the classical treatment. The limits of the abscissa are $N \chi_{\text {crit }}$ and $N \chi_{s}$ corresponding to $\phi_{0}=0.16$. 
between the coexistence curve and the spinodal curve and vary $N \chi$. We have performed numerical calculations for two compositions $\phi_{0}=0.16$ and $\phi_{0}=0.1$. These compositions are chosen so as to be far away from the critical region where meanfield theory is inapplicable. ${ }^{14}$ They also coincide with the compositions used in the recent experiment by Balsara and co-workers. ${ }^{8}$ Since the results for these two compositions are qualitatively similar, detailed presentation and discussion of the results will be for $\phi_{0}=0.16$ only. We will, however, make use of the free energy data calculated for $\phi_{0}=0.1$ when we discuss the experiments.

For $\phi_{0}=0.16$, the metastable region is bounded by $(N \chi)_{\text {coex }}=2.43857$ at the coexistence curve and $(N \chi)_{s}$ $=3.72023$ at the spinodal. Obviously, the thermodynamically stable phase in coexistence with $\phi_{0}=0.16$ has $\phi_{0}$ $=0.84$.

For each given $N \chi$, the density profile of the nucleus is determined by solving the self-consistent field equations subject to a specified value of the density of $A$ polymers at some particular radial distance, as mentioned in the last section. The resulting profile is then used to obtain a new guess of the density at the specified radius, until the profile obtained is sufficiently close to that of the critical nucleus. From the density profile $\phi(r)$, we define the material excess, $M^{\text {ex }}$,

$$
M^{\mathrm{ex}} \equiv 4 \pi \int_{0}^{\infty} r^{2} d r\left[\phi(r)-\phi_{0}\right],
$$

which measures the excess amount of $A$ polymers relative to the (metastable) bulk composition $\phi_{0}=0.16$. In this definition, $M^{\mathrm{ex}}$ has the dimensions of volume, and measures the volume taken by the excess $A$ polymers in a given nucleus. The grand potential is obtained from Eq. (2.4) and the free energy of formation of the nucleus is calculated from Eq. (2.3). The critical nucleus is identified as the nucleus corresponding to a free energy maximum with respect to the material excess, $M^{\text {ex }}$, as illustrated in Fig. 1. We comment that the free energy of formation at $M^{\mathrm{ex}}=0$ vanishes as it should; thus our calculation is free of the consistency problems encountered in classical capillary theory. ${ }^{1}$

To make the results applicable for general $N$, it is convenient to use dimensionless radial distance $\widetilde{r}$, material excess $\tilde{M}^{\text {ex }}$, and free energy of formation $\Delta \widetilde{F}$ defined as

$$
\begin{aligned}
& \tilde{r} \equiv \frac{r}{N^{1 / 2} b}, \\
& \tilde{M}^{\mathrm{ex}} \equiv \frac{M^{\mathrm{ex}}}{N^{3 / 2} b^{3}}, \\
& \Delta \tilde{F} \equiv \frac{\beta \Delta F}{N^{1 / 2}} \frac{v}{b^{3}} .
\end{aligned}
$$

Near the coexistence curve, the critical nucleus is expected to approach that given by the classical approximation in which a sharp interface separates a large nucleus from the parent bulk phase. As the spinodal is approached, the CahnHilliard asymptotic theory predicts that the critical nucleus density profile becomes large in extent but small in amplitude. In Fig. 3, we show three representative density profiles for the critical nucleus calculated at three values of the $N \chi$

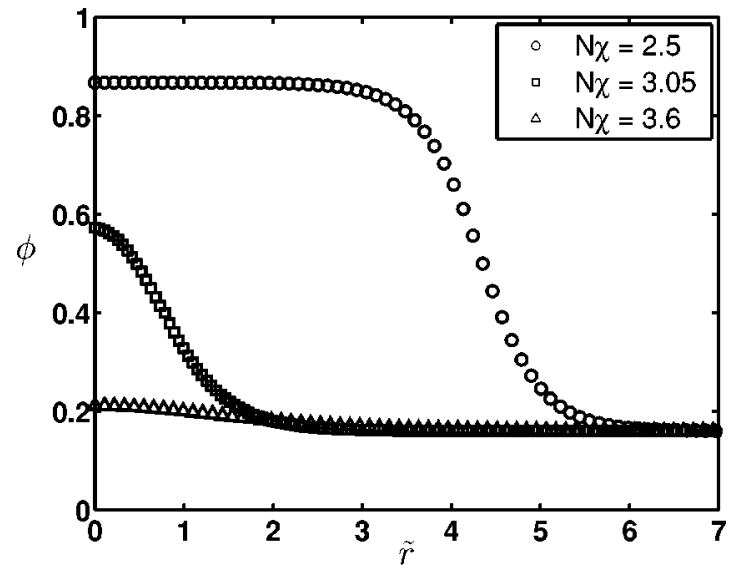

FIG. 3. Three representative density profiles of the critical nucleus for $\phi_{0}$ $=0.16$. For this choice of $\phi_{0}, N \chi_{\text {coex }}=2.43857$, and $N \chi_{s}=3.72023$.

parameter, one close to the coexistence curve, one close to the spinodal, and one intermediate between the coexistence curve and the spinodal. As can be seen from the figure, for $N \chi=2.5$, the density profile is fairly flat up to a distance of 3 times the end-to-end distance of the chain. Thus the interior of the nucleus can be considered a pseudophase with nearly uniform density. However, the interfacial width for this value of $N \chi$ is not small and is nearly comparable to the size of the uniform core. Nevertheless, when the correct interfacial tension is used, the classical theory gives predictions that are in good agreement with results obtained using the SCF theory.

As $N \chi$ increases toward the spinodal, the density profile of the critical nucleus becomes more diffuse. For $N \chi$ $=3.05$, it is no longer possible to distinguish between the core and the interfacial regions. The density of $A$ at the center of the nucleus is now considerably lower than the equilibrium value of the new phase. As $N \chi$ increases further to 3.6, the profile becomes very shallow with the density at the center only slightly exceeding that of the parent bulk phase. Upon careful inspection, one can see that the spatial range of the density variation for $N \chi=3.6$ is larger than that for $N \chi$ $=3.05$.

The variation of the density of $A$ at the center of the critical nucleus as $N \chi$ increases is shown in Fig. 2. Also shown on the same figure are the coexistence curve and spinodal curves. The circles at a constant $\phi_{0}=0.16$ and varying values of $N \chi$ specify the bulk condition of the metastable phase and the squares are the density of $A$ at the center of the critical nucleus corresponding to each $N \chi$. Interestingly, very close to the coexistence curve, the center density of the critical nucleus slightly exceeds that of the equilibrium coexistence value. This behavior can be understood by noting that near the coexistence curve the core of the critical nucleus can be considered to be at pseudo "phase coexistence" with the metastable bulk. Since the chemical potential of $A$ in the metastable bulk is higher than its equilibrium value, the density of $A$ in the new "phase" is higher than the equilibrium coexistence value. Of course, as $N \chi$ approaches the coexistence curve, the size of the critical nucleus diverges, and we approach a true phase coexistence between 


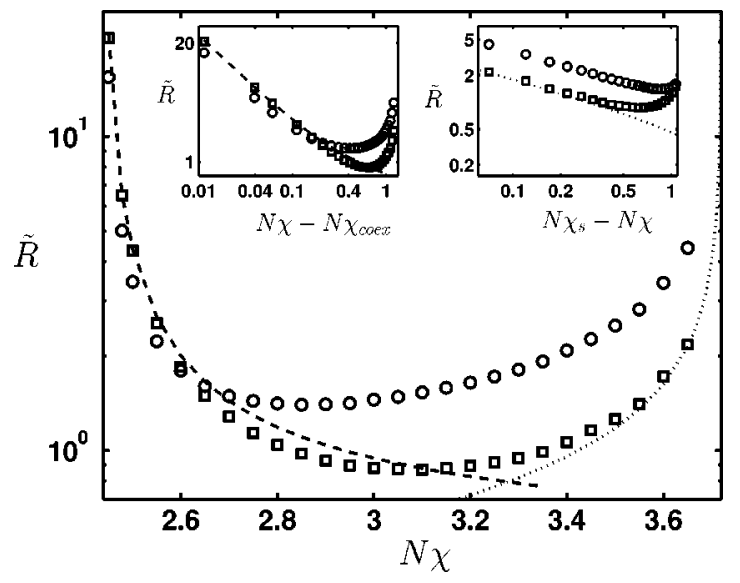

FIG. 4. Radius of the critical nucleus as defined by the first moment (circles), and by $\widetilde{R}_{1 / 2}$ (squares). The limits on the abscissa correspond to $N \chi_{\text {coex }}$ and $N \chi_{s}$. Included are the $\widetilde{R}_{1 / 2}$ results of Cahn and Hilliard near the spinodal (dotted line) and of the classical treatment near the coexistence curve (dashed line).

the critical nucleus and the bulk; the density in the nucleus becomes identical to the equilibrium density of the new phase. As $N \chi$ increases from the coexistence curve, the center density first increases and then decreases, eventually approaching the bulk value $\phi_{0}$ at the spinodal, as dictated by consistency.

The radius of the critical nucleus is well defined in the classical limit, but becomes ambiguous far from the coexistence curve, essentially losing its meaning as the spinodal is approached (Fig. 3). To provide a measure of the spatial extent of the nucleus, we present our results using two alternate definitions of radius: the first moment of the density profile of the nucleus, defined as

$$
R_{1} \equiv \frac{4 \pi}{M^{\mathrm{ex}}} \int_{0}^{\infty} r^{3} d r\left[\phi(r)-\phi_{0}\right],
$$

and the half-peak radius $R_{1 / 2}$ used by Cahn and Hilliard, defined as the radius at which

$$
\phi(r=0)-\phi\left(R_{1 / 2}\right)=\phi\left(R_{1 / 2}\right)-\phi_{0} .
$$

The dependence of these two defined radii on $N \chi$ is shown in Fig. 4. Both of these definitions give the same scaling dependence on $N \chi-(N \chi)_{\text {coex }}$ and $(N \chi)_{s}-N \chi$ near the coexistence curve and spinodal, respectively (insets), although the numerical values obtained from the two definitions can differ by as much as a factor of 2.5 near the spinodal. The minima of these two quantities occur at different values of $N \chi$. The two definitions approach each other near the coexistence curve, as both give a measure of the size of the uniform droplet. The deviation between these two measures thus indicates the breakdown of the picture of the nucleus as a uniform droplet. Near the coexistence curve and spinodal, the radius defined by both measures diverge, in agreement with the Cahn-Hilliard predictions obtained using a square gradient approach. The classical nucleation theory, which is not thermodynamically consistent near the spinodal, predicts that the radius of the critical nucleus approaches a nonzero constant in that limit. ${ }^{4}$

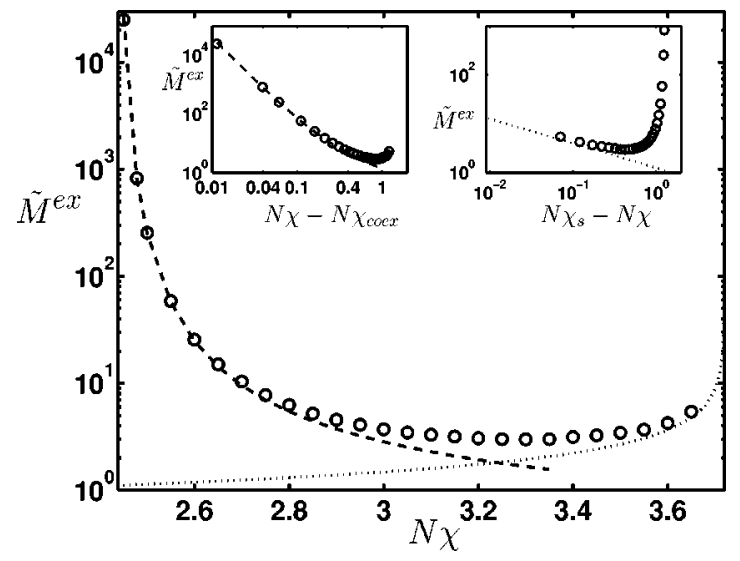

FIG. 5. Material excess in the critical nucleus as function of $N \chi$. The limits on the abscissa correspond to $N \chi_{\text {coex }}$ and $N \chi_{s}$. Included are the results of Cahn and Hilliard near the spinodal (dotted line) and of the classical treatment near the coexistence curve (dashed line).

The material excess $M^{\text {ex }}$ serves as a fundamental characterization of a nucleating cluster and can be considered the order parameter for nucleation. ${ }^{18}$ In the present case, $M^{\text {ex }}$ is directly related to the number of polymer chains involved in forming the critical nucleus. Since $M^{\text {ex }}$ is the volume taken by the excess $A$ polymer chains in the nucleus, and each polymer chain has volume $N v$, the excess number of $A$ polymers is

$$
n_{A}^{\mathrm{ex}}=\frac{N^{1 / 2} b^{3}}{v} \tilde{M}^{\mathrm{ex}}
$$

Because $\tilde{M}^{\text {ex }}$ depends only on the bulk composition $\phi_{0}$ and $N \chi$ of the metastable phase, for a given $\phi_{0}$ and $N \chi$, the number of excess $A$ polymers increases as $N^{1 / 2}$. The dimensionless material excess $\widetilde{M}^{\text {ex }}$ is shown as a function of $N \chi$ in Fig. 5. Like the radius of the critical nucleus, this quantity exhibits a minimum intermediate between the coexistence curve and the spinodal and diverges in these two limits. The minima of $\tilde{M}^{\text {ex }}$ and critical nucleus radius occur at different $N \chi$, again reflecting the nonuniformness of the density in the critical nucleus.

The most important quantity concerning the critical nucleus is the reversible work of formation. This free energy provides the activation barrier for the formation of the new phase from a metastable phase and is directly related to the rate of the kinetics of this process. The free energy barrier diverges at the coexistence curve, in agreement with the prediction by classical theory, and as demanded by thermodynamic consistency. As $N \chi$ increases away from the coexistence curve, the free energy barrier decreases, reflecting the fact that the metastable phase becomes less stable. The free energy barrier vanishes at the spinodal, in spite of the divergence of both the radius and the material excess. This is again demanded by thermodynamic consistency, since by its very definition, at the spinodal a system is unstable with respect to small, long length scale perturbation. The work of formation is shown on a log-linear scale in Fig. 6 in order to reveal the large order of magnitude changes as $N \chi$ increases. The free energy decreases rapidly near the coexistence curve and then undergoes a relatively slow decrease between $N \chi$ 


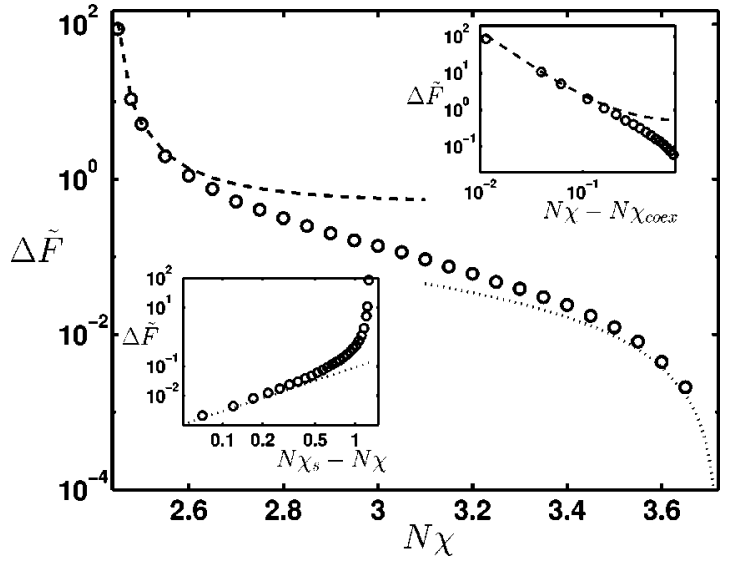

FIG. 6. Free energy of formation of the critical nucleus as function of $N \chi$. The limits on the abscissa correspond to $N \chi_{\text {coex }}$ and $N \chi_{s}$. Included are the results of Cahn and Hilliard near the spinodal (dotted line) and of the classical treatment near the coexistence curve (dashed line).

$\approx 2.6$ and $N \chi \approx 3.4$. As $N \chi$ approaches the spinodal, the free energy again decreases rapidly, eventually to zero at the spinodal. We will see that the classical theory and Cahn-Hilliard asymptotic theory are valid in the two respective narrow regions near the coexistence curve and spinodal; however, the broad range of $N \chi$ cannot be described by these two limiting theories.

\section{B. Comparison with the classical theory and the Cahn-Hilliard asymptotic theory}

Our self-consistent field calculation provides an exact description of the critical nuclei at the mean-field level. With the results we have obtained for the large range of $N \chi$ between the coexistence curve and the spinodal, we can now evaluate the accuracy of the two limiting theories.

In the classical theory, the nucleus is taken to be a spherical droplet of uniform density separated by a sharp interface from the parent metastable bulk phase. The work of formation of the droplet is assumed to consist of two contributions, an excess free energy for the interior of the droplet and the interfacial free energy; thus

$$
\Delta F=\frac{4 \pi R^{3}}{3}\left(g-g_{0}\right)+4 \pi R^{2} \gamma,
$$

where $\gamma$ is the interfacial tension and $g$ is the grand potential per unit volume,

$$
g\left(\phi, \mu_{0}\right)=f(\phi)-\mu_{0} \phi,
$$

with $\mu_{0}$ the chemical potential of the metastable phase, $\mu_{0}$ $=\left.(\partial f / \partial \phi)\right|_{\phi_{0}}$. The density in the nucleus is determined from $\partial g / \partial \phi=0$. Using the free energy of the blend for a homogenous state given in the Appendix, we obtain

$$
\begin{aligned}
\ln \phi & -\ln (1-\phi)+N \chi(1-2 \phi) \\
& =\ln \phi_{0}-\ln \left(1-\phi_{0}\right)+N \chi\left(1-2 \phi_{0}\right),
\end{aligned}
$$

where the reader is reminded that $\chi$ here stands for $\chi v$ in the Appendix. Obviously, one solution of Eq. (3.10) is $\phi=\phi_{0}$, which simply reaffirms that the metastable state is a minimum of the free energy $g$. Another solution, with the density

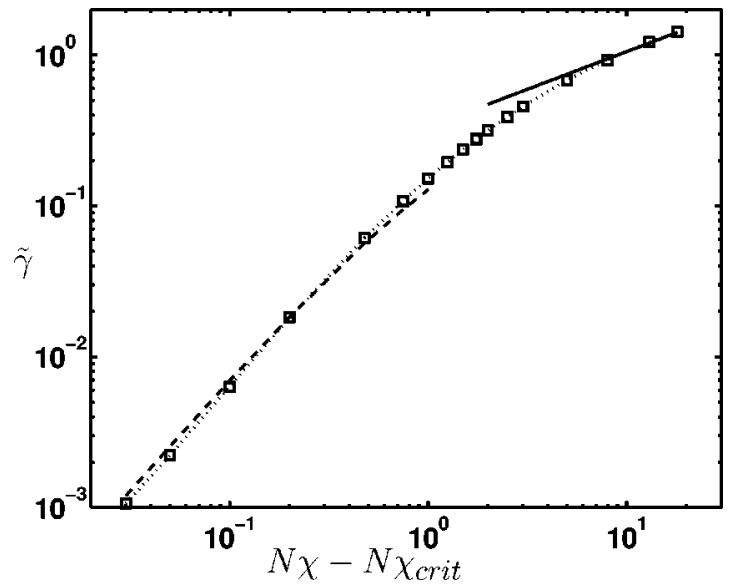

FIG. 7. Interfacial free energy vs $\left(N \chi-N \chi_{\text {crit }}\right)$. The squares show data obtained in this work. The solid curve shows the behavior predicted by de Gennes (Ref. 15) for large $N \chi$; the dashed curve shows the behavior predicted by Joanny and Leibler (Ref. 31) for $N \chi$ near the critical point value; and the dotted curve shows the polynomial fit through data from this work used for calculating the interfacial contribution to the nuclei in the classical theory. The dimensional interfacial free energy $\gamma$ is obtained from $\bar{\gamma}$ by $\gamma$ $=k_{B} T N^{-1 / 2}(b / v) \bar{\gamma}$.

falling within the spinodal region of the phase diagram, corresponds to a free energy maximum, and therefore cannot be the density of the nucleus of the new phase. Only the remaining solution gives the physical solution for the density of the nucleus, and hence the free energy $g$ of the nucleus. Note that $g-g_{0}<0$, since the free energy of the newly formed phase is lower than that of the metastable state.

The critical nucleus is that for which $\partial F / \partial R=0$, which yields

$$
R=2 \frac{\gamma}{g_{0}-g} .
$$

This corresponds to a free energy barrier of

$$
\Delta F=\frac{16 \pi}{3} \frac{\gamma^{3}}{\left(g_{0}-g\right)^{2}} .
$$

The material excess for the classical model is simply

$$
M^{\mathrm{ex}}=\frac{4 \pi R^{3}}{3}\left(\phi-\phi_{0}\right)
$$

For the interfacial free energy, we use the interfacial tension between the two coexisting phases at the $N \chi$ of interest. Since an analytical expression is not available except near the critical point and for very large $N \chi$, we have performed a separate SCF calculation to determine the interfacial tension as a function of $N \chi$. The data are shown in Fig. 7 for a large range of $N \chi$ values. A polynomial interpolation is used to obtain the numerical value of $\gamma$ for a given $N \chi$.

The predictions of the classical theory for the density of the critical nucleus $\phi$, the radius $R$, the material excess $M^{\text {ex }}$, and the work of formation $\Delta F$ are shown in the respective figures where the self-consistent field results are presented. Clearly, near the coexistence curve, good agreement is observed between results from the classical theory and those from the SCF calculation. This is so even though the interfacial width in the range of $N \chi$ considered is rather diffuse 
on the scale of the size of the polymer. Of these quantities, the agreement for $M^{\mathrm{ex}}$ is particularly remarkable since it covers a fairly broad region away from the coexistence curve. However, this apparent agreement at larger values of $N \chi$ is likely due to a cancellation of errors. For example, at $N \chi$ $=2.8$ both $\phi$ and $R$ deviate considerably from the SCF results, yet good agreement is still obtained for $M^{\text {ex }}$. The free energy provides a more stringent comparison. The curve predicted from the classical theory and that predicted from the SCF begin to diverge from each other visibly at about $N \chi$ $=2.6$.

The approach of Cahn and Hilliard ${ }^{4}$ employed a free energy functional where the nonlocal terms were approximated by a square gradient term. The general framework they provided is valid for any binary mixture. They also obtained numerical data throughout the metastable region of the phase diagram for an incompressible binary mixture using a model free energy. However, explicit application of their data for the present polymer blend problem is not possible except in the region close to the spinodal where they provided analytical expressions. We thus compare our SCF results with the Cahn-Hilliard results in this limit.

For an incompressible binary polymer blend, an expression for the square gradient term was given by de Gennes. ${ }^{15}$ Near the spinodal, the density gradient in the critical nucleus is small and the square gradient approximation is valid. For the local term, we use the free energy density derived in the Appendix. The free energy functional is then

$$
\begin{aligned}
\Delta F= & \int d \mathbf{r}\left[\left(f[\phi(\mathbf{r})]-f\left(\phi_{0}\right)\right)-\mu_{0}\left(\phi(\mathbf{r})-\phi_{0}\right)\right. \\
& \left.+\frac{b^{2}}{36 v \phi(1-\phi)}(\nabla \phi(\mathbf{r}))^{2}\right] .
\end{aligned}
$$

Near the spinodal, the volume fraction $\phi$ in the critical nucleus is close to the metastable bulk value $\phi_{0}$. Thus Cahn and Hilliard expanded the free energy density $f[\phi(\mathbf{r})]$ as a power series in $\Delta \phi \equiv \phi-\phi_{0}$ and kept terms up to $(\Delta \phi)^{3}$. The results of their analyses were expressed in terms of the difference of the bulk composition from its spinodal value at a given temperature (or $N \chi$ in our case). Experimentally, it is much more convenient to vary $\chi$ (through changes in temperature or pressure) at a fixed composition. We thus present the results as a function of $1-\chi / \chi_{s}$. After some straightforward substitution of notation, the reversible work of formation of the critical nucleus is found to be

$$
\beta \Delta F=2.23 \frac{N^{1 / 2} b^{3}}{v} \frac{\phi_{0}\left(1-\phi_{0}\right)}{\left(1-2 \phi_{0}\right)^{2}}\left(1-\frac{\chi}{\chi_{s}}\right)^{3 / 2} .
$$

The density at the center of the nucleus is

$$
\phi(r=0)-\phi_{0}=8.1 \frac{\phi_{0}\left(1-\phi_{0}\right)}{1-2 \phi_{0}}\left(1-\frac{\chi}{\chi_{s}}\right) .
$$

The value of $R_{1 / 2}$ is given by

$$
R_{1 / 2}=0.3 N^{1 / 2} b\left(1-\frac{\chi}{\chi_{s}}\right)^{-1 / 2} .
$$

And finally, the material excess in the nucleus is ${ }^{19}$

$$
M^{\mathrm{ex}}=3.3 N^{3 / 2} b^{3} \frac{\phi_{0}\left(1-\phi_{0}\right)}{1-2 \phi_{0}}\left(1-\frac{\chi}{\chi_{s}}\right)^{-1 / 2} .
$$

Note that in these equations, it is assumed that $\phi_{0}<0.5$; otherwise $\phi_{0}$ should be exchanged with $1-\phi_{0}$.

Predictions using these asymptotic expressions are compared with results from our SCF in the plots for the corresponding properties. The density at the center of the critical nucleus agrees remarkably well with the SCF result, extending quite far from the spinodal. On the other hand, deviations are significant for the radius, the material excess and the free energy barrier, aside from the few data points near the spinodal. Calculation using the SCF becomes difficult as the spinodal is approached because of numerical instabilities and critical slowing down. On the other hand, the asymptotic expressions should become exact in this limit (since both the square gradient approximation and the order parameter expansion truncated at the cubic order become increasingly accurate as the spinodal is approached). Therefore, these asymptotic predictions provide an excellent extrapolation for our SCF results near the spinodal.

Comparison between the result of our SCF calculation for the free energy barrier and the predictions by the two limiting theories shows that, over a broad range of $N \chi$ between the coexistence curve and the spinodal, the two limiting theories do not provide an accurate prediction. It is within this range that our SCF is most valuable.

\section{Validity of the SCF theory: Application to experiments}

The SCF theory is a mean-field theory which neglects thermal fluctuations. Within the mean-field framework, a clear distinction exists between nucleation and spinodal decomposition, with the two mechanisms separated by the spinodal curve. In the metastable region, as the spinodal is approached, thermal fluctuations become increasingly important; at the same time, the free energy barrier $\Delta F$ for nucleation decreases. The barrier is no longer meaningful when $\Delta F \sim k T$. The condition $\Delta F \sim k T$ signals the breakdown of the mean-field approximation and can be considered a Ginzburg criterion for the validity of mean-field theory in the metastable region. ${ }^{5}$ Within the Ginzburg region, it is no longer possible to distinguish between nucleation and spinodal decomposition. No satisfactory theories exist in this crossover region, which is characterized by nonclassical exponents and fractal-like structures for the incipient phase. ${ }^{20,21}$ A correct theoretical treatment would require a kinetic ${ }^{22}$ rather than thermodynamic approach.

To rigorously address the validity of the mean-field approach requires that fluctuation effects be taken into account explicitly in the expression for the rate of nucleation. Such a framework was developed by Langer ${ }^{23}$ some time ago, who included fluctuation contributions at the quadratic level near the saddle point of the free energy surface. While this program can in principle be carried out in the present study, incorporation of fluctuation around an inhomogeneous saddle-point state for polymeric systems is highly nontrivial $^{24}$ and is beyond the scope of this paper. We will therefore settle for using the free energy barrier calculated 
from the SCF as a quantitative Ginzburg criterion. Following Binder $^{5}$ we set the threshold at $\Delta F=10 k T$. Thus we propose that mean-field theory breaks down when

$$
\beta \Delta F \equiv \frac{N^{1 / 2} b^{3}}{v} \Delta \widetilde{F}=10 .
$$

For a given degree of polymerization $N$ and composition $\phi_{0}$ in the metastable region, the above equation determines the value of $N \chi$ or $\chi$-denoted as $(N \chi)_{G}$ and $\chi_{G}$, respectively, at which mean-field theory becomes invalid. For the composition used in our SCF study $\phi_{0}=0.16$, if we take $N$ $=100,10^{3}$, and $10^{4}$, we estimate $(N \chi)_{G}$ to be $2.79,3.05$, and 3.34, respectively, corresponding to $\chi_{G} / \chi_{s}=0.75,0.82$, and 0.90 . For general compositions, the width of the Ginzburg region $1-\chi_{G} / \chi_{s}$ can be obtained approximately using the Cahn-Hilliard asymptotic expression Eq. (3.15) which yields

$$
1-\frac{\chi_{G}}{\chi_{s}}=2.72 \frac{v^{2 / 3}}{b^{2}} \frac{\left(1-2 \phi_{0}\right)^{4 / 3}}{\left[\phi_{0}\left(1-\phi_{0}\right)\right]^{2 / 3}} N^{-1 / 3} .
$$

Because the Cahn-Hilliard asymptotic theory is valid only very close to the spinodal, the above estimate is accurate only for large $N$. For moderate $N$, the width of the Ginzburg region predicted by the asymptotic theory is wider than that predicted by the SCF since the asymptotic theory underestimates the free energy barrier (see Fig. 6).

We now discuss our results in connection to the recent experiment by Balsara and co-workers. ${ }^{8}$ The experiment used a binary blend made of partially deuterated polymethylbutylene (A) and hydrogeneous polyethylbutylene (B), with degrees of polymerization $N_{A}=3357$ and $N_{B}=4260$, respectively. Two compositions were studied: $\phi_{0}=0.161$ (sample B1) and $\phi_{0}=0.099$ (sample B2). The work reports unusual behavior in the early stage of nucleation in the ranges $0.84<\chi^{\prime} \chi_{s} \approx 1$ for sample B1 (including one data point at $\left.\chi^{\prime} \chi_{s}>1\right)$ and $0.7<\chi / \chi_{s}<1$ for sample B2. In particular they find that the size of the critical nucleusidentified as the inverse of the wave vector at which the scattering intensity remains unchanged-increases as $\chi$ is decreased toward the coexistence curve and has an extrapolated divergence at about $\chi^{/} \chi_{s} \approx 0.81$ and 0.68 for $\mathrm{B} 1$ and $\mathrm{B} 2$, respectively. These findings are in clear contradiction with all known theoretical predictions.

Because the two polymers studied in Ref. 8 are not symmetric, application of our SCF results calculated for a symmetric blend to the experimental system is not immediately obvious. However, the effect of the molecular asymmetry can be incorporated explicitly into the Cahn-Hilliard asymptotic theory, with results that appear rather similar to Eqs. (3.15)-(3.18). In particular, the free energy barrier can be shown to be

$\beta \Delta F=2.23(1+\delta)^{2} \frac{V_{\mathrm{eff}}^{1 / 2}}{l_{\mathrm{eff}}^{3 / 2}} \frac{\phi_{0}\left(1-\phi_{0}\right)}{\left(1-2 \phi_{0}\right)^{2}}\left(1-\frac{\chi}{\chi_{s}}\right)^{3 / 2}$,

where

$$
V_{\mathrm{eff}}=\frac{V_{A} V_{B}}{V_{A} \phi_{0}+V_{B}\left(1-\phi_{0}\right)},
$$

$$
\delta=\frac{\phi_{0}\left(1-\phi_{0}\right)\left(V_{A}-V_{B}\right)}{V_{B}\left(1-\phi_{0}\right)^{2}-V_{A} \phi_{0}^{2}},
$$

and

$$
l_{\mathrm{eff}}=\frac{l_{A} l_{B}}{l_{A} \phi_{0}+l_{B}\left(1-\phi_{0}\right)},
$$

with $l_{\alpha} \equiv V_{\alpha} / R_{\alpha}^{2}(\alpha=A, B)$. In these equations, $V_{\alpha}$ and $R_{\alpha}^{2}$ are, respectively, the molecular volume and mean square end-to-end distance of the $\alpha$ chains. We use these quantities since they are experimentally more accessible than $N_{\alpha}, v_{\alpha}$, and $b_{\alpha}$.

We see that near the spinodal, the main effect of the molecular asymmetry, aside from a correction factor (1 $+\delta)^{2}$, is the replacement of the overall free energy scale $N^{1 / 2} b^{3} / v$ by $V_{\text {eff }}^{1 / 2} / l_{\text {eff }}^{3 / 2}$. We will assume that this remains true as long as the relative distance from the spinodal is not too large, so that the free energy barrier for the asymmetric case can be simply obtained from that for the symmetric case by using Eq. (3.4) with the proper rescaling by this factor.

Reference 8 suggests $V_{A}=335700 \AA^{3}, \quad V_{B}$ $=426000 \AA^{3}$. From the radii of gyration of the two polymers given in the same reference, we infer $l_{A}=2.19 \AA, l_{B}$ $=2.77 \AA$. The factor $V_{\text {eff }}^{1 / 2} / l_{\text {eff }}^{3 / 2}$ changes little $(\approx 1 \%)$ between the two compositions; its average value is 174 . The correction factor $(1+\delta)^{2}$ varies between 1.04 at $\phi_{0}=0.099$ and 1.08 at $\phi_{0}=0.161$; given the approximations involved and the error margins in the parameters used, we will ignore this factor. By the same token, we ignore the small differences between the experimental compositions and the compositions we used in the SCF study $\left(\phi_{0}=0.161\right.$ versus 0.16 and $\phi_{0}=0.099$ versus 0.10 ).

Keeping in mind these caveats, we estimate the onset of the Ginzburg region to be at $\chi_{G} / \chi_{s}=0.86$ for $\phi_{0}=0.16$ and $\chi_{G} / \chi_{s}=0.80$ for $\phi_{0}=0.1$, using a threshold free energy barrier of $10 k T$. Thus for both compositions, the width of the Ginzburg region is quite significant, considering the reasonably large degrees of polymerization (by experimental standards). At $\chi^{\prime} \chi_{s}=0.84$, the first data point in the experiment at $\phi_{0}=0.16$, we estimate the free energy barrier to be $15 \mathrm{kT}$. For $\chi^{\prime} \chi_{s}=0.7$ at $\phi_{0}=0.1$, the free energy barrier is $24 k T$. At $\chi^{\prime} \chi_{s}=0.81$ and 0.68 corresponding to the extrapolated divergence of the size of the critical nuclei for samples B1 and $\mathrm{B} 2$, we estimate the free energy barrier to be $23 k T$ and $29 k T$, respectively.

Given that the $\chi$ range in which the data were reported in Ref. 8 overlaps with the Ginzburg region we have identified here, it is possible that the findings of Lefebvre et al. reveal new dynamic behavior in the phase separation kinetics when the free energy barrier becomes of order $k T$. In the Ginzburg region, the kinetics cannot be unambiguously described as either nucleation or spinodal decomposition, but rather reflects a crossover between these two mechanisms. On the other hand, the spinodal is no longer meaningful when the Ginzburg region is not small. The location of the mean-field spinodal, and the determination of the $\chi$ parameter itself, become problematic in this case. ${ }^{25}$ Although the spinodal in Ref. 8 was identified with great care, ${ }^{26}$ one cannot rule out the possibility that the location of the "apparent spinodal" 
could be shifted due to the large fluctuation; thus the kinetics studied in the experiments could be more of the nature of spinodal decomposition than nucleation. Indeed, it has been suggested that the onset of the Ginzburg region should be taken as the kinetic spinodal that separates the physically accessible metastable region from the unstable region. ${ }^{27,28}$ The evidence that the researchers in Ref. 8 were indeed observing nucleation was strong but not overwhelming. That we find a moderate free energy barrier $(23 k T$ and $29 k T$ for $\phi_{0}=0.16$ and 0.1 , respectively) at the $\chi / \chi_{s}$ value where the size of the critical nucleus showed extrapolated divergence in the experiment could also be due to possible uncertainties in the experimental determination of $\chi$ and/or $\chi_{s}$. For the conclusions of Ref. 8 to be definitive, it would be desirable to perform systematic experiments with $\chi^{\prime} \chi_{s}$ less than 0.81 and 0.68 for samples B1 and B2. The work of Lefebvre et al. reported one shallow quench experiment on B2 at $\chi / \chi_{s}$ $=0.57$ and found no evidence of nucleation for 1000 mins. At this value of $\chi$, our SCF calculation predicts a free energy barrier of $144 k T$. This is a prohibitively high free energy barrier for nucleation to occur.

Cahn and Hilliard suggested a free energy barrier of $60 k T$ for an observable nucleation rate in small-molecule binary fluid mixture. ${ }^{4}$ Given that relaxation dynamics in polymeric fluids is slower than in small-molecule fluids, we anticipate that a somewhat smaller free energy barrier is required for nucleation to be observable. In the absence of a kinetic theory for nucleation in polymers, we will use $60 \mathrm{kT}$ as the upper limit of the free energy barrier. For $\phi_{0}=0.16$, our SCF results determine that this free energy barrier corresponds to $N \chi=2.8$, or $\chi / \chi_{s}=0.75$. For $\phi_{0}=0.1$, we obtain $N \chi=3.5$ or $\chi / \chi_{s}=0.62$. Thus, we suggest that systematic experiments be performed for $\chi / \chi_{s}>0.75$ at $\phi_{0}=0.16$, and for $\chi^{\prime} \chi_{s}>0.62$ at $\phi_{0}=0.1$. Experimental data from such a systematic study would clarify the nature of the process occurring in this metastable region (nucleation versus spinodal decomposition), and help determine the limit of validity of the mean-field approximation where nucleation occurs.

\section{CONCLUSION}

Using self-consistent field theory, we have studied various properties of the critical nucleus for a symmetric binary polymer blend, spanning the entire metastable region of the phase diagram from the coexistence curve to spinodal at a fixed composition. At the mean-field level, the self-consistent field theory represents the most accurate and systematic theory that fully accounts for the chain connectivity and the spatial correlations that result from it. Thus it provides a useful benchmark for evaluating approximate theories, such as the classical capillary theory and the square gradient density functional theory.

Our results show that the near the coexistence curve, the critical nucleus is large and of nearly uniform density. The composition of the bulk minority component in the nucleus is slightly higher than the equilibrium composition of the new phase. As the controlling parameter (in our case $N \chi$ ) progresses toward the spinodal, the density of the bulk minority component decreases, and eventually becomes indistinguishable from the bulk density at the spinodal. The work of formation of the critical nucleus representing the free energy barrier for nucleation starts from infinity at the coexistence curve, decreases rapidly away from the coexistence curve and vanishes at the spinodal. On the other hand, both the radius of the critical nucleus and the material excess in it exhibit a minimum somewhere intermediate between the coexistence curve and the spinodal, and diverge at the two limits. All these predictions are in qualitative agreement with earlier predictions of Cahn and Hilliard ${ }^{4}$ using a square gradient density functional theory.

We have provided a detailed comparison between predictions of the SCF study and the classical nucleation theory near the coexistence curve on one hand and the CahnHilliard asympotic theory near the spinodal on the other. We find good agreement between the SCF results and these two limiting theories in their respective limits. However, the agreement for most properties is limited to narrow regions near these two limits. In particular, the free energy barrier in a wide range of $N \chi$ cannot be well represented by these limiting theories.

We have examined the validity of the SCF theory by invoking a Ginzburg criterion using the free energy barrier. We find that for typical degrees of polymerization, the Ginzburg region is not as small as commonly believed. In particular, we find that some of the experimental data reported in the recent work of Lefebvre et al. ${ }^{8}$ are either in the Ginzburg region or near it. The theoretical implication of their experimental findings is not entirely clear. Their results may reflect new dynamic behavior in the phase separation kinetics when the free energy barrier becomes of order $k T$. It is also possible that thermal fluctuation leads to a shift in the location of the apparent spinodal. If we take the onset of the Ginzburg criterion as the physical or kinetic spinodal, then some of the data points in Ref. 8 are already beyond this spinodal. The predicted relative location of the kinetic spinodals for the two compositions is consistent with the experimental results. Finally, nucleation in polymeric systems may involve mechanisms that are fundamentally different from those envisioned in a quasiequilibrium approach.

Outside the Ginzburg region, our theory provides a quantitative prediction for the free energy barrier for nucleation which can be related to the nucleation rate. We estimate that a window of $\chi^{\prime} \chi_{s}$ exists in which the SCF is valid and at the same time the nucleation rate is not too small. Thus it will be desirable that experiments be performed in this window to provide a clear test of the theory. We remark that in this window, neither the classical theory nor the CahnHilliard asymptotic theory gives an accurate prediction of the free energy barrier for typical degrees of polymerization. The agreement in the free energy barrier between the CahnHilliard asymptotic theory and our SCF is limited to a narrow region near the spinodal that is contained in the Ginzburg region for moderately long chains. Thus, while it provides a simple means of determining the validity of meanfield theory, in practice there is no regime where the CahnHilliard asymptotic theory is quantitatively valid except for very long polymers. On the other hand, the classical capillary theory, while valid and accurate near the coexistence curve, is irrelevant as far as quantitative prediction of the nucleation 
rate is concerned, since the nucleation rate is vanishingly small for polymers near the coexistence curve.

In future work, we plan to study the effects of polydispersity and impurities on nucleation. Binder pointed out ${ }^{3}$ that in a polydisperse blend, the long chains may serve as preferential centers for nucleation, so in effect we have heterogeneous rather than homogeneous nucleation. Evidence ofheterogeneous nucleation was shown in the work of Cumming et al., ${ }^{29}$ although no explanation was provided for the origin of such behavior. Another extension of the theory is to study the effects of diblock copolymers made from the two homopolymers. Earlier experiments by Balsara and coworkers on nucleation in polymer blends in fact involved a small amount of diblock copolymers. ${ }^{6,7}$ However, the effects of the diblocks are not clear. On one hand, addition of these amphiphilic molecules should facilitate nucleation because they lower the interfacial energy by adsorption at the $A / B$ interface; on the other hand, addition of diblock copolymers alters the phase behavior of the blend in a way that decreases the thermodynamic driving force for phase separation. Thus we expect richer and more interesting nucleation behavior in such a 3-component system.

\section{ACKNOWLEDGMENTS}

This work is supported in part by the National Science Foundation (DMR-9970589) and the Camille and Henry Dreyfus Foundation (TC-96-063). The authors thank Professor Nitash Balsara for sending us a preprint of their work Ref. 8 and for helpful discussions.

\section{APPENDIX: DERIVATION OF THE SCF EQUATIONS}

In this appendix, we provide a detailed derivation of the self-consistent field equations for an open system of an incompressible polymer blend. The derivation is similar to that by Matsen ${ }^{30}$ for a homopolymer-copolymer blend. The SCF equations are used to solve for the density profile and calculate the free energy of the critical nucleus. In addition, the solution of these SCF equations for a homogeneous state provides the free energy density used in the classical theory and the square-gradient theory.

We start with the statistical mechanical equivalent of the free energy [Eq. (2.1)], the grand partition function for an open, incompressible system of a two component polymer blend,

$$
\begin{aligned}
& \Xi(\mu, V, T) \equiv \exp [-\beta G]=\sum_{n_{A}=0}^{\infty} \sum_{n_{B}=0}^{\infty} \frac{1}{n_{A} ! n_{B} !} \frac{1}{\left(N_{A} v_{A}\right)^{n_{A}}\left(N_{B} v_{B}\right)^{n_{B}}} \exp \left(\beta \mu n_{A} N_{A} v_{A}\right) \int D^{n_{A}}\left\{\mathbf{R}_{A}\right\} \\
& \times \int D^{n_{B}}\left\{\mathbf{R}_{B}\right\} \prod_{\mathbf{r}} \delta\left(\hat{\phi}_{A}+\hat{\phi}_{B}-1\right) \exp \left[-\beta \sum_{i=1}^{n_{A}} h_{i}^{A}-\beta \sum_{j=1}^{n_{B}} h_{j}^{B}-\chi \int d \mathbf{r} \hat{\phi}_{A}(\mathbf{r}) \hat{\phi}_{B}(\mathbf{r})\right] .
\end{aligned}
$$

Here, $\beta \equiv 1 / k T, v_{\alpha}$ and $N_{\alpha}$ are, respectively, the monomer volume and the degree of polymerization of the $\alpha$ polymer; $h_{i}^{\alpha}$ is the single chain Hamiltonian for the $i$ th polymer of species $\alpha$ that accounts for the chain connectivity; and $\hat{\phi}_{\alpha}(\mathbf{r})$ is the instantaneous volume fraction of $\alpha$ at $\mathbf{r}$ defined as $\hat{\phi}_{\alpha}(\mathbf{r})=v_{\alpha} \hat{\rho}_{\alpha}(\mathbf{r})$ with $\hat{\rho}_{\alpha}(\mathbf{r})$ the instantaneous density. The last term in the exponential represents the local repulsive interaction between the two polymers and the notation $\int D^{n} \alpha\left\{\mathbf{R}_{\alpha}\right\}$ represents integration over all chain configurations (including the center of mass translation) of polymers of type $\alpha$. Note that we have used the chain volume $N_{\alpha} v_{\alpha}$ as the volume scale in the partition function instead of the cube of the thermal de Broglie wavelength; the effect is simply a constant (i.e., composition independent) shift in the chemical potential which has no consequences on the thermodynamics of interest. tity

Introducing collective variables $\phi_{\alpha}(\mathbf{r})$ through the iden-

$$
\prod_{\mathbf{r}} \int D \phi_{\alpha} \delta\left(\phi_{\alpha}-\hat{\phi}_{\alpha}\right)=1,
$$

and using the Fourier representation of the $\delta$-function, we can rewrite the partition function as a multi-fold functional integral,

$$
\begin{aligned}
\Xi(\mu, V, T)= & \int D \phi_{A} \int D \phi_{B} \int D W_{A} \int D W_{B} \\
& \times \prod_{\mathbf{r}} \delta\left(\phi_{A}+\phi_{B}-1\right) \\
& \times \exp \left[-\beta K\left(\phi_{A}, \phi_{B}, W_{A}, W_{B}\right)\right],
\end{aligned}
$$

where

$$
\begin{aligned}
\beta K= & \chi \int d \mathbf{r} \phi_{A} \phi_{B}-i \int d \mathbf{r}\left(W_{A} \phi_{A}+W_{B} \phi_{B}\right) \\
& +\beta I\left[W_{A}, W_{B}\right] .
\end{aligned}
$$

The integration over the auxiliary fields $W_{\mathrm{A}}(\mathbf{r})$ and $W_{\mathrm{B}}(\mathbf{r})$ results from the Fourier representation of the $\delta$-function in Eq. (A2), and $I$ is defined through

$$
\begin{aligned}
\exp (-\beta I)= & \sum_{n_{A}=0}^{\infty} \sum_{n_{B}=0}^{\infty} \frac{1}{n_{A} ! n_{B} !} \frac{1}{\left(N_{A} v_{A}\right)^{n_{A}}\left(N_{B} v_{B}\right)^{n_{B}}} \\
& \times \exp \left(\beta \mu n_{A} N_{A} v_{A}\right) Z_{A}^{n_{A}} Z_{B}^{n_{B}} \\
= & \exp \left[\frac{\exp \left(\beta \mu N_{A} v_{A}\right) Z_{A}\left(W_{A}\right)}{N_{A} v_{A}}+\frac{Z_{B}\left(W_{B}\right)}{N_{B} v_{B}}\right],
\end{aligned}
$$


where $Z_{A}$ and $Z_{B}$ are the single chain configurational integrals in the presence of external fields of magnitude $i\left(k_{B} T\right) v_{A} W_{A}$ and $i\left(k_{B} T\right) v_{B} W_{B}$, respectively. Taking the polymer chains to be Gaussian, we have

$$
\begin{aligned}
Z_{\alpha}\left(W_{\alpha}\right)= & \int D \mathbf{r}_{\tau} \exp \left(\frac{-3}{2 b_{\alpha}^{2}} \int_{0}^{N_{\alpha}} d \tau\left(\frac{d \mathbf{r}_{\tau}}{d \tau}\right)^{2}\right. \\
& \left.-i v_{\alpha} \int_{0}^{N_{\alpha}} d \tau W_{\alpha}\left(\mathbf{r}_{\tau}\right)\right) \\
= & \int d \mathbf{r} q_{\alpha}\left(\mathbf{r}, N_{\alpha}\right),
\end{aligned}
$$

where $q_{\alpha}\left(\mathbf{r}, N_{\alpha}\right)$ is a reduced partition function for a chain with one end at $\mathbf{r}$ and the other end anywhere and is determined by the following diffusion equation:

$$
\left(\frac{\partial}{\partial \tau}-\frac{b_{\alpha}^{2}}{6} \nabla_{\mathbf{r}}^{2}+i v_{\alpha} W_{\alpha}(\mathbf{r})\right) q(\mathbf{r} ; \tau)=0,
$$

with initial condition $q(\mathbf{r} ; 0)=1$. Because the system is incompressible, the local composition is uniquely specified by either $\phi_{A}$ or $\phi_{B}=1-\phi_{A}$. We denote $\phi_{A}$ simply by $\phi$; the integration over $\phi_{B}$ in Eq. (A3) can be trivially performed to yield

$$
\begin{aligned}
\Xi(\mu, V, T)= & \int D \phi \int D W_{A} \int D W_{B} \\
& \times \exp \left[-\beta K\left(\phi, W_{A}, W_{B}\right)\right]
\end{aligned}
$$

with now

$$
\begin{aligned}
\beta K= & \chi \int d \mathbf{r} \phi(1-\phi)-i \int d \mathbf{r}\left(W_{A} \phi+W_{B}(1-\phi)\right) \\
& +\beta I\left[W_{A}, W_{B}\right] .
\end{aligned}
$$

The development thus far is exact. We now make the self-consistent field approximation which amounts to evaluating the functional integral, Eq. (A8), by its value at the stationary point (saddle point) of the functional $K\left(\phi, W_{A}, W_{B}\right)$. The value of $K$ at the saddle point is then equated with the grand potential $G$. Setting the functional derivatives of $K$ with respect to its variables to zero produces the set of self-consistent field equations given in Eqs. (2.7)(2.9). The saddle point values of $W_{\alpha}$ turn out to lie on the imaginary axis; thus we have defined $w_{\alpha}=i W_{\alpha}$ so that all the variables are now real. It should be understood that the field variables $\phi, w_{A}$, and $w_{B}$ appearing in the SCF equations refer to their saddle point values. However, for simplicity, we do not introduce any new notation.

The SCF equations [Eqs. (2.7)-(2.9)] can be easily solved for the case of a homogenous state with uniform composition. For the rest of this derivation, homogeneity is understood; in the body of the paper, values of variables for the homogeneous case are denoted with subscript 0 . For spatially independent $\phi, w_{A}$, and $w_{B}$, the SCF equations become

$$
\begin{aligned}
& w_{A}-w_{B}=\chi(1-2 \phi), \\
& \phi=\exp \left(\beta \mu N_{A} v_{A}-w_{A} N_{A} v_{A}\right), \\
& 1-\phi=\exp \left(-w_{B} N_{B} v_{B}\right) .
\end{aligned}
$$

It is more convenient to write the solutions in terms of $w_{A}, w_{B}$, and $\mu$ as functions of the composition $\phi$; doing so we obtain

$$
\begin{aligned}
& w_{A}=\chi(1-2 \phi)-\frac{1}{N_{B} v_{B}} \ln (1-\phi), \\
& w_{B}=-\frac{1}{N_{B} v_{B}} \ln (1-\phi),
\end{aligned}
$$

and

$$
\beta \mu=\frac{1}{N_{A} v_{A}} \ln \phi-\frac{1}{N_{B} v_{B}} \ln (1-\phi)+\chi(1-2 \phi) .
$$

These results can be substituted into Eq. (2.4) to yield the grand potential per unit volume as

$$
\beta g=\frac{1}{N_{B} v_{B}} \ln (1-\phi)-\frac{\phi}{N_{A} v_{A}}-\frac{1-\phi}{N_{B} v_{B}}+\chi \phi^{2},
$$

from which we obtain the Helmholtz free energy density,

$$
\begin{aligned}
\beta f(\phi)= & \frac{\phi}{N_{A} v_{A}}[\ln \phi-1]+\frac{1-\phi}{N_{B} v_{B}}[\ln (1-\phi)-1] \\
& +\chi \phi(1-\phi) .
\end{aligned}
$$

Noting that the free energy of mixing is simply

$$
f_{\text {mix }}=f(\phi)-[\phi f(\phi=1)+(1-\phi) f(\phi=0)],
$$

we recover the celebrated Flory-Huggins free energy,

$$
\beta f_{\text {mix }}=\frac{\phi}{N_{A} v_{A}} \ln \phi+\frac{1-\phi}{N_{B} v_{B}} \ln (1-\phi)+\chi \phi(1-\phi) .
$$

${ }^{1}$ P. G. Debenedetti, Metastable Liquids: Concepts and Principles (Princeton University Press, Princeton, 1996).

${ }^{2}$ D. W. Oxtoby, in Fundamentals of Inhomogeneous Fluids, edited by Douglas Henderson (Dekker, New York, 1992), pp. 407-442.

${ }^{3}$ K. Binder, J. Chem. Phys. 79, 6387 (1983).

${ }^{4}$ J. W. Cahn and J. E. Hilliard, J. Chem. Phys. 31, 688 (1959).

${ }^{5}$ K. Binder, Phys. Rev. A 29, 341 (1984).

${ }^{6}$ N. P. Balsara, C. Lin, and B. Hammouda, Phys. Rev. Lett. 77, 3847 (1996).

${ }^{7}$ A. A. Lefebvre, J. H. Lee, H. S. Jeon, N. P. Balsara, and B. Hammouda, J. Chem. Phys. 111, 6082 (1999).

${ }^{8}$ A. A. Lefebvre, J. H. Lee, N. P. Balsara, and B. Hammouda, J. Chem. Phys. (submitted).

${ }^{9}$ K. M. Hong and J. Noolandi, Macromolecules 14, 727 (1981).

${ }^{10}$ G. J. Fleer, M. A. Cohen Stuart, J. M. H. M. Scheutjens, T. Cosgrove, and B. Vincent, Polymers at Interfaces (Chapman and Hall, London, 1993).

${ }^{11}$ E. Helfand, J. Chem. Phys. 62, 999 (1975).

${ }^{12}$ M. W. Matsen and M. Schick, Phys. Rev. Lett. 72, 2660 (1994).

${ }^{13}$ D. W. Oxtoby, Acc. Chem. Res. 31, 91 (1998).

${ }^{14}$ P.-G. de Gennes, J. Phys. (France) Lett. 38, L-441 (1977).

${ }^{15}$ P.-G. de Gennes, J. Chem. Phys. 72, 4756 (1980).

${ }^{16}$ A. Z. Akcasu and I. C. Sanchez, J. Chem. Phys. 88, 7847 (1988).

${ }^{17}$ H. Tang and K. F. Freed, J. Chem. Phys. 94, 1572 (1991); 94, 6321 (1991); X. C. Zeng, D. W. Oxtoby, H. Tang, and K. F. Freed, ibid. 96, 4816 (1992)

${ }^{18}$ I. Kusaka, Z.-G. Wang, and J. H. Seinfeld, J. Chem. Phys. 108, 3416 (1998); I. Kusaka and D. W. Oxtoby, ibid. 110, 5249 (1999).

${ }^{19}$ The numerical prefactor has been approximated from a graphic integration of the dimensionless density profile provided in Ref. 4.

${ }^{20}$ D. W. Heermann and W. Klein, Phys. Rev. B 27, 1732 (1983).

${ }^{21}$ W. Klein and C. Unger, Phys. Rev. B 28, 445 (1983); C. Unger and W. Klein, ibid. 29, 2698 (1984). 
${ }^{22}$ G. Narsimhan and E. Ruckenstein, J. Colloid Interface Sci. 128, 549 (1989); E. Ruckenstein and B. Nowakowski, ibid. 137, 583 (1990).

${ }^{23}$ J. S. Langer, Ann. Phys. (N.Y.) 54, 258 (1969); in Systems Far From Equilibrium, edited by L. Garrido (Springer-Verlag, Heidelberg, 1980), pp. 12-47.

${ }^{24}$ For a treatment of the equilibrium fluctuation in ordered diblock copolymer phases, see A.-C. Shi, J. Noolandi, and R. C. Desai, Macromolecules 29, 6487 (1996)

${ }^{25}$ Z.-G. Wang (unpublished).

${ }^{26}$ A. A. Lefebvre, J. H. Lee, N. P. Balsara, B. Hammouda, R. Krishnamoorti, and S. Kumar, Macromolecules 32, 5460 (1999); A. A. Lefebvre, J. H. Lee, N. P. Balsara, and B. Hammouda, ibid. 33, 7977 (2000).

${ }^{27}$ A. Z. Patashinskii and B. I. Shumilo, Sov. Phys. Solid State 22, 655 (1980).

${ }^{28}$ S. B. Kiselev and I. G. Kostyukova, J. Chem. Phys. 98, 6455 (1993).

${ }^{29}$ A. Cumming, P. Wiltzius, F. S. Bates, and J. H. Rosedale, Phys. Rev. A 45, 885 (1992).

${ }^{30}$ M. W. Matsen, Phys. Rev. Lett. 74, 4225 (1995).

${ }^{31}$ J. F. Joanny and L. Leibler, J. Phys. (Paris) 39, 951 (1978). 